Cahiers d'études japonaises

19 | 2012

Le Japon et le fait colonial II

\title{
Rôle et pouvoir des éditorialistes dans la presse du milieu de l'ère Meiji (1884-1894). Fukuzawa Yukichi, Kuga Katsunan, Tokutomi Sohō
}

\section{Frank Ackerer}

\author{
(2) OpenEdition \\ Journals \\ Édition électronique \\ URL : https://journals.openedition.org/cipango/1740 \\ DOI : $10.4000 /$ cipango. 1740 \\ ISSN : 2260-7706 \\ Éditeur \\ INALCO \\ Édition imprimée \\ Date de publication : 30 octobre 2012 \\ Pagination : 261-267 \\ ISBN : 978-2-85831-204-7 \\ ISSN : $1164-5857$
}

Référence électronique

Frank Ackerer, «Rôle et pouvoir des éditorialistes dans la presse du milieu de l'ère Meiji (1884-1894). Fukuzawa Yukichi, Kuga Katsunan, Tokutomi Sohō », Cipango [En ligne], 19 | 2012, mis en ligne le 15 mai 2014, consulté le 30 juin 2021. URL : http://journals.openedition.org/cipango/1740 ; DOI : https:// doi.org/10.4000/cipango.1740

Ce document a été généré automatiquement le 30 juin 2021.

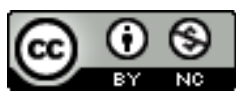

Cipango est mis à disposition selon les termes de la Licence Creative Commons Attribution - Pas d'Utilisation Commerciale 4.0 International. 


\title{
Rôle et pouvoir des éditorialistes dans la presse du milieu de l'ère Meiji (1884-1894). Fukuzawa Yukichi, Kuga Katsunan, Tokutomi Sohō
}

\author{
Frank Ackerer
}

\section{RÉFÉRENCE}

Frank ACKERER, Rôle et pouvoir des éditorialistes dans la presse du milieu de l'ère Meiji (1884-1894). Fukuzawa Yukichi, Kuga Katsunan, Tokutomi Sohō, thèse de doctorat soutenue sous la direction d'Emmanuel Lozerand, INALCO, 2012, 552 p.

1 Les premières décennies de l'ère Meiji sont le théâtre de nombreux conflits. Au plan de la politique intérieure, les oligarques arrivés au pouvoir lors de la restauration de 1868 sont confrontés, à partir du milieu des années 1870, à une opposition qui s'organise progressivement et réclame le droit de participer à l'élaboration des décisions. Sous la pression du Mouvement pour la liberté et les droits du peuple, les autorités s'engagent en 1881 à mettre en place un parlement pour l'année 1890 au plus tard. Cette promesse ouvre une période riche en débats sur les contours du régime à venir, mais aussi sur les objectifs et les moyens de la diplomatie japonaise. La perspective de l'entrée en vigueur prochaine d'une monarchie constitutionnelle suscite, notamment, l'espoir d'obtenir enfin la révision des traités inégaux imposés par les puissances occidentales à partir de 1858.

2 Dans le foisonnement des débats entourant les questions de politique étrangère, les éditorialistes de la presse d'opinion sont en première ligne. Pourtant, leur influence est souvent négligée. Les historiens de l'ère Meiji ont produit de nombreux ouvrages sur la 
presse, ainsi que des études très fouillées sur les négociations entourant la révision des traités, mais rien ou presque sur l'influence effective des éditorialistes dans le processus de négociation. La vision dominante cantonne généralement la presse de l'ère Meiji à un rôle de spectateur (remuant, certes, mais spectateur quand même). Or, les années 1880 voient apparaitre de nombreux organes, quotidiens ou revues qui, s'ils ne sont pas liés aux partis politiques, revendiquent néanmoins une participation active aux grands débats de l'heure. Née de la volonté de mieux saisir le rôle de cette presse d'opinion indépendante, cette thèse s'est fixé pour objectif d'évaluer précisément l'influence des éditorialistes dans le processus de décision politique entre 1884 et 1894.

3 Notre démarche se situe à la croisée de deux disciplines. L'importance du contexte, la nécessité de dresser des chronologies fines de l'action politique imposaient une approche historique. En même temps, le besoin de caractériser l'argumentation et le positionnement des éditorialistes nous obligeait à accorder une place centrale à l'analyse des textes. Alors que l'histoire politique d'une part, celle des idées ou des médias de l'autre, restent assez souvent sur des traces parallèles, nous avons tenté ici de croiser les approches afin de mesurer véritablement ce que pouvait être l'action par les mots.

4 La période étudiée débute en 1884, quand de grands quotidiens à visées commerciales et capables d'exprimer une opinion indépendante prennent le pas, en termes de tirage, sur les journaux liés aux partis politiques, et se termine en 1894, à la veille de la guerre opposant le Japon à la Chine. 1884 est également l'année de la révolte des paysans endettés du Chichibu, dont l'échec a pour conséquence de faire apparaître la violence collective comme une voie définitivement sans espoir. Si l'on interprète le déclenchement de la guerre sino-japonaise comme le moment où la violence commence à s'exercer vers l'extérieur, il est possible de considérer que la décennie 1884-1894 consacre le renoncement à la violence dans la politique intérieure, renoncement dont la montée en puissance des éditorialistes peut être vue comme la conséquence.

$5 \mathrm{Au}$ moment de définir notre corpus, la diplomatie s'est imposée comme un thème privilégié. Si l'on excepte la "fenêtre» de Nagasaki, le Japon a été presque complètement fermé au monde pendant plus de deux siècles, et l'insertion du pays dans le système international place les autorités face à des problèmes complexes. La révision des traités inégaux devient l'une des préoccupations essentielles du pouvoir politique, et donc l'un des principaux enjeux du débat public, à partir du milieu des années 1880 , quand cet objectif commence à sembler accessible.

6 Enfin, nous avons choisi de limiter notre étude à l'activité de trois figures de la presse d'opinion : Fukuzawa Yukichi 福澤諭吉 (1835-1901), Kuga Katsunan 陸羯南 (1857-1907) et Tokutomi Sohō 徳富蘇峰 (1863-1957). Les sources primaires principales, constituées d'éditoriaux rédigés par ces trois intervenants, représentent un corpus de près de 400 articles. Notre démonstration s'est appuyée sur environ un quart de ces textes; pour la plupart, ils sont présentés en traduction intégrale. En exploitant ce corpus, il s'agissait de dégager la posture de chacun des éditorialistes, par rapport au gouvernement et à l'opinion publique, et donc de fournir un point d'appui pour l'analyse de leur action politique effective.

7 La thèse est divisée en deux parties. Le premier travail a consisté à caractériser l'argumentation de Fukuzawa, Kuga et Tokutomi. Fukuzawa fait partie de «l'ancienne génération ", celle qui a fait ses études pendant les dernières années du shôgunat, et qui a vu avec des yeux d'adulte la pression militaire exercée par les Occidentaux à 
partir du milieu des années 1850. Il fonde dès 1882 ce qui est généralement considéré comme le premier grand quotidien d'opinion indépendant : Les Nouvelles de notre temps (Jiji shinpō 時事新報). Introducteur inlassable de la pensée occidentale au Japon, c'est un libéral pour qui le contact avec les Occidentaux ne peut que faire progresser la société et l'économie japonaises. Kuga est l'un des plus actifs représentants de la "jeune génération ». Formé comme Fukuzawa à l'étude des classiques chinois dans son enfance, il a ensuite étudié, notamment le droit français, dans les nouveaux établissements mis en place après la restauration. Ce qu'il perçoit dans les années 1880 comme une occidentalisation à outrance du Japon l'inquiète. Si ce juriste de formation considère que nombre d'idées ou de techniques occidentales sont susceptibles d'être introduites dans la société japonaise avec profit, il craint que cela n'entraîne la dilution, voire la désintégration de l'identité nationale. Celui que l'historiographie décrit souvent comme le premier représentant du nationalisme japonais fonde le quotidien Le Japon (Nihon 日本) en 1889. Quant à Sohō, il est le cadet de Kuga de seulement six ans, et partage avec lui le fait d'avoir reçu une éducation reposant sur deux piliers distincts. D'abord formé aux classiques chinois, il s'initie ensuite à la pensée anglo-saxonne en recevant l'enseignement de missionnaires chrétiens. Sohō est un jeune homme optimiste qui s'engage à partir de 1887, à travers sa revue L'Ami du peuple (Kokumin no tomo 国民之友), pour l'installation de la démocratie au Japon. Il fondera trois ans plus tard, en 1890, le quotidien Le Journal du peuple (Kokumin shinbun 国民新聞).

L'étude conjointe de ces trois intellectuels a permis de cerner la figure de l'éditorialiste, qui apparaît à cette époque, et de comprendre son rôle, et plus largement celui de la presse, dans la formation de l'espace public moderne au Japon. En même temps, elle a fait émerger des spécificités nettes. Une analyse détaillée des textes, faisant appel aux concepts de la théorie de l'argumentation, a mis en évidence la préférence de Fukuzawa pour les arguments pragmatiques et les métaphores, le goût de Kuga pour la polémique et les démonstrations rigoureuses, reposant sur des arguments juridiques aussi bien qu'économiques et la tendance de Sohō à privilégier les prises de position idéologiques. Les ressources discursives de l'écriture éditorialiste sont donc multiples, et trois images émergent de ce travail d'analyse : un Fukuzawa pédagogue et prêt au compromis, un Kuga combatif et rigoureux dans ses démonstrations, enfin un Sohō peu enclin à s'engager dans des débats concrets. Afin de préciser ces images, une attention particulière a été portée à l'utilisation que fait chacun de la notion d'«opinion publique ». Fiction du droit constitutionnel légitimant la parole du journaliste chez Fukuzawa et Kuga, l'opinion publique recouvre dans la pensée de Sohō les opinions amalgamées d'un groupe vaste, mais abstrait que le journaliste a la responsabilité de guider. À partir de ces conclusions, un éditorial-type a été défini pour chacun des trois éditorialistes.

9 La deuxième partie de la thèse est consacrée à l'étude de trois crises diplomatiques ayant fortement marqué de leur empreinte l'histoire politique du Japon de l'ère Meiji, en un moment clé de son émancipation politique le conduisant à se dégager du carcan des traités inégaux. Il s'agit du naufrage du Normanton, en 1886, de la crise politique de 1889 débouchant sur l'attentat dont est victime le ministre des Affaires étrangères ōkuma, et de l'incident d'Ōtsu, au cours duquel le policier Tsuda tente de s'en prendre à la vie du tsarévitch Nicolas, en visite officielle au Japon en 1891. Si l'affaire du Normanton et l'incident d'ōtsu ne durent que quelques semaines, la crise de l'année 1889 s'étend sur plus de six mois. À l'aide des outils mis au point dans la première partie (caractérisation des argumentations, définition d'éditoriaux-types, 
utilisation du concept d'« opinion publique »), l'activité des éditorialistes est analysée, puis mise en regard de façon minutieuse avec l'évolution du positionnement des autorités. Cette confrontation fait apparaître, dans le processus de gestion gouvernementale de chaque crise, des inflexions qu'il est possible de relier sans ambiguïté à la pression exercée par la presse d'opinion. Autrement dit, les éditorialistes parviennent à imposer des changements de cap significatifs : révision d'un jugement du tribunal consulaire anglais que les autorités avaient initialement accepté sans broncher (1886), remise en cause de négociations diplomatiques bien engagées, alors que les États-Unis, l'Allemagne et la Russie avaient déjà signé le projet proposé par la diplomatie japonaise (1889) et mise en échec d'une tentative d'ingérence gouvernementale dans le processus de décision judiciaire (1891).

Trois conclusions essentielles émergent de notre analyse. Premièrement, par rapport aux images véhiculées par l'historiographie, ce travail modifie notre regard sur Kuga et Tokutomi, tandis qu'il confirme notre perception de Fukuzawa. En effet, le portrait que dessinent les éditoriaux du Jiji shinpō n'est pas surprenant; l'auteur a été beaucoup étudié et ses positions sont connues. Simplement, ce travail permet de l'observer dans son activité d'éditorialiste, « au quotidien ». Il s'agit d'un éclairage relativement inédit, car si de nombreux spécialistes ont parcouru les volumes 1 à 7 de ses œuvres complètes (volumes comprenant l'autobiographie, les écrits sur l'Occident, l'Appel à l'étude...), les volumes 8 à 16, qui regroupent les éditoriaux, avaient été jusqu'ici peu exploités. Kuga, loin de l'image du nationaliste agressif brossée par certains commentateurs, impressionne ici par sa compétence. Ses articles sont clairs, bien documentés. L'auteur montre une excellente connaissance du droit, mais aussi des rouages de l'économie, ainsi qu'un goût prononcé pour les arguments faisant appel aux chiffres. Il déjoue les classifications attendues par les spécialistes des nationalismes européens, en ce qu'il est tout à la fois un nationaliste conséquent et un pacifiste rigoureux. Tokutomi est probablement celui pour lequel le résultat de notre analyse est le plus surprenant. L'enthousiasme provoqué par des ouvrages de jeunesse comme Le Japon à venir (1886) contraste étrangement avec le manque de conviction des éditoriaux consacrés aux questions de diplomatie. Comment l'expliquer? Il semble que Tokutomi, dans les années 1884-1894, concentre son énergie sur une réforme de la société qu'il juge plus cruciale que l'amélioration de la position internationale du Japon. En outre, la fadeur de ses arguments tient sans doute également dans le fait qu'il n'est pas un journaliste au sens strict, mais plutôt un essayiste, et que le rythme imposé par le format de l'éditorial ne lui convient pas réellement.

11 Deuxièmement, dans leur effort en vue de participer à la prise de décision politique, on constate que les éditorialistes ne manquent pas d'armes. Le concept d'opinion publique représente pour eux un « levier » essentiel, et les circuits de circulation de la parole qui se mettent progressivement en place permettent à leurs arguments d'être largement relayés. Nous avons montré dans cette étude que l'idée d'opinion publique était présente dans les esprits dès la première décennie de l'ère Meiji, et que les éditorialistes s'y référaient très tôt dans le but de «lester » leurs prises de position. À mesure que l'idée d'un régime représentatif s'impose dans le débat, cette notion acquiert une importance croissante. Au milieu de la décennie 1880-1890, le concept d'opinion publique devient à la fois central (il correspond à la légitimité dont se réclament ceux qui ont des lecteurs et non des partisans) et problématique, puisqu'il reçoit des acceptions différentes d'un auteur à l'autre. Fukuzawa et Kuga en font une utilisation consciente et relativement sophistiquée. Alors que, selon leur conception, 
l'opinion publique résulte de débats entre individus «éclairés », l'enjeu, pour les deux éditorialistes, consiste à présenter cette "opinion publique " comme un groupe beaucoup plus large. En ayant notamment recours au procédé de la communion avec les lecteurs, Fukuzawa se place toujours à l'intérieur du lectorat et, au-delà, de l'opinion publique qu'il affirme représenter. Kuga, bien qu'il soit objectivement le porte-parole d'une élite intellectuelle et économique, cherche à s'en dissocier en se posant en défenseur de ceux "qui aiment le Japon». L'opinion publique qu'invente l'éditorialiste du Nihon est donc celle des " patriotes » (aikokusha 愛国社). Quant à Sohō, il considère que l'opinion publique n'est pas à même de percevoir la réalité du jeu politique, et qu'elle doit s'en remettre aux journalistes pour se faire une opinion. Sa théorisation du rôle du journaliste le place donc au-dessus d'une opinion publique abstraite, qu'il lui revient de guider.

On pourrait supposer que les discours analysés ne concernent que les lecteurs de la capitale, mais l'étude des journaux de province, au moment de l'affaire du Normanton, nous a permis d'identifier une importante diffusion des arguments de Fukuzawa dans les différentes régions du pays. Au milieu des années 1880, la plupart des journaux de province sont encore jeunes, et leur santé financière ne leur permet pas de recruter des correspondants. De plus, un véritable système de distribution nationale des journaux n'est pas encore en place. C'est pourquoi il est possible d'observer, jusqu'aux années 1890, une démultiplication des arguments des éditorialistes à l'échelon national, au moins dans le cas de Fukuzawa. Ces années correspondent à un "moment" particulier, celui où, grâce au développement de la circulation de la parole, quelques éditorialistes semblent créer une « opinion publique » faisant contrepoids à un pouvoir qui, jusque-là, n'avait de comptes à rendre à personne.

13 Troisièmement, l'émergence d'un véritable débat public, dans les années 1880 , nous amène à nuancer la vision communément admise des formes du pouvoir politique durant l'ère Meiji. L'historiographie emploie souvent l'expression "gouvernements d'oligarques ", et le choix de ces termes laisse croire que les hommes au pouvoir prennent les décisions essentielles en toute autonomie. Or, notre travail a montré, au moins pour la décennie 1884-1894, que le pouvoir est en réalité disputé, partagé, et que les éditorialistes, sur les questions de diplomatie, participent pleinement au processus de décision en infléchissant la politique étrangère du gouvernement. Bien souvent, la décision émerge de débats triangulaires impliquant le cabinet, les éditorialistes et l'« opinion publique ». La qualité de l'argumentation des éditorialistes, leur capacité à s'affirmer comme des représentants de l'« opinion publique » ainsi que la coïncidence de leur lectorat avec la classe des «décideurs» constituent incontestablement trois éléments permettant d'expliquer cette forme de partage du pouvoir. Il convient donc de procéder à une réévaluation de l'influence des éditorialistes, réévaluation qui conduit, par voie de conséquence, à relativiser la prééminence du gouvernement dans la conduite des affaires du pays. Avant même l'ouverture du Parlement, le régime a commencé à changer de nature. Ce constat permet de faire un sort - s'il fallait encore le faire - à cette idée simple selon laquelle le Japon serait devenu une démocratie après 1945, suite aux réformes imposées par l'occupant américain. 